\title{
Contrast-enhanced ultrasound and real-time elastography in the differential diagnosis of malignant and benign thyroid nodules
}

\author{
XIN SUI ${ }^{1}$, HUAI-JUN LIU ${ }^{2}$, HONG-LI JIA ${ }^{3}$ and QIN-MAO FANG ${ }^{1}$ \\ ${ }^{1}$ Department of Ultrasound, The Third Hospital of Hebei Medical University, Shijiazhuang, Hebei 050051; \\ ${ }^{2}$ Department of Radiology, The Second Hospital of Hebei Medical University, Shijiazhuang, Hebei 050050; \\ ${ }^{3}$ Department of Radiology, The First Hospital of Hebei Medical University, Shijiazhuang, Hebei 050051, P.R. China
}

Received March 10, 2015; Accepted January 26, 2016

DOI: $10.3892 / \mathrm{etm} .2016 .3344$

\begin{abstract}
The diagnostic value of contrast-enhanced ultrasound (CEUS) or real-time elastography (RTE) alone, as well as a combination of CEUS and RTE, in distinguishing benign from malignant thyroid nodules was investigated. Between August 2012 and June 2014, a total of 97 consecutive patients (50 male and 47 female patients; mean age, $48.6 \pm 12.4$; age range, 27-70 years) with thyroid nodules referred for surgical treatment were examined by CEUS and RTE. The final diagnosis was obtained based on histological findings. Image analysis of the CEUS and RTE scans was performed. Considering the postoperative pathological results as the golden standard, a receiver operating characteristic (ROC) curve was constructed. Subsequently, the sensitivity, specificity, positive predictive value (PPV), negative predictive value (NPV) and accuracy of CEUS alone, RTE alone and CEUS + RTE combination were calculated. Pathological examination showed 66 papillary carcinomas and 43 benign lesions, including 21 adenomas and 22 nodular goiters. The sensitivity, specificity, PPV, NPV and accuracy of CEUS were 81.82, 90.70, 93.10, 90.70 and $85.32 \%$, respectively. In the case of RTE, the sensitivity, specificity, PPV, NPV and accuracy were 80.30, $88.37,91.38,88.37$ and $83.49 \%$, respectively. Furthermore, the combination of CEUS + RTE had a sensitivity of $95.45 \%$, specificity of $95.35 \%$, PPV of $96.92 \%$, NPV of $95.35 \%$ and accuracy of $95.41 \%$. Therefore, the CEUS + RTE combination showed a significantly higher sensitivity and specificity compared with CEUS or RTE alone (all $\mathrm{P}<0.05$ ). Based on ROC analysis, the area under the curve (AUC) for CEUS, RTE and CEUS + RTE combination was 0.883, 0.863 and 0.959, respectively. The AUC of RTE alone was significantly lower
\end{abstract}

Correspondence to: Professor Huai-Jun Liu, Department of Radiology, The Second Hospital of Hebei Medical University, 215 Heping West Road, Shijiazhuang, Hebei 050050, P.R. China E-mail: liuhuaijun_lhj@163.com

Key words: ultrasound, real-time elastography, contrast-enhanced ultrasound, pathological examination, malignant and benign thyroid nodules, differential diagnosis compared with that of the CEUS + RTE combination. In conclusion, our results demonstrate that CEUS + RTE combination significantly increases the diagnostic performance for differential diagnosis of malignant and benign thyroid nodules compared with CEUS or RTE alone.

\section{Introduction}

Thyroid nodules are commonly benign and the reported prevalence widely varies depending on the population studied and the methods used to detect the nodules (1). Globally, thyroid cancer is increasing rapidly and resulted in 36,000 fatalities in 2010, an increase from 24,000 in 1990, although 5 year survival rates are high following treatment (2-4). A previous study state that, between 1992 and 2006, a total of 43,644 thyroid cancer cases wee diagnosed in the United States (5). In China, thyroid cancer is the 8th most frequent cancer, and the rapid increase in thyroid cancer incidence represents a substantial health burden (6,7). Ultrasound (US) is an accepted standard diagnostic method for the detection of thyroid nodules worldwide (8). However, previous studies found extreme variations in the assessment of thyroid nodules by US (9-11). Conventional US has a moderate accuracy for characterizing the nature of thyroid nodules, thus supplementary diagnostic methods, including radionuclide scanning and fine-needle aspiration (FNA) biopsy, are employed for improved clinical evaluation of thyroid nodules (12-14). With the introduction of contrast-enhanced ultrasound (CEUS) and real-time elastography (RTE), promising results have been reported for better accuracy in differentiating between benign and malignant thyroid nodules (15-17).

CEUS involves the use of a contrast medium that enhances the diagnostic imaging capabilities of traditional medical sonography and is a milestone for diagnostics in liver tumors, with recent studies evaluating similar application of CEUS in characterizing thyroid gland tumors (18-23). In addition, CEUS performed with the use of a microbubble contrast agent may be a potentially useful adjunct in assessing thyroid nodules, since it has a high specificity of $84.8 \%$ and high sensitivity of $76.9 \%$ (21). CEUS is also amenable to combination approaches, and dynamical evaluation of microcirculation with CEUS in combination with color-coded and power Doppler sonography was demonstrated to yield reliable preoperative results in the 
characterization of thyroid adenomas (22). Furthermore, the combination of CEUS with acoustic radiation force impulse (ARFI) has been shown to significantly improve the diagnostic accuracy of thyroid nodules (23). As a noninvasive technique for evaluating thyroid nodules, US-based elastography encompasses a variety of approaches, including RTE, acoustic radiation force impulse imaging and supersonic shear imaging (24). RTE is an imaging technique that can directly reveal the physical properties of tissues through the use of conventional US probes. Elastography was recently demonstrated to be an invaluable diagnostic method in differentiating between various pathologies in 283 patients with Hashimoto's thyroiditis $(16,25)$. A strain index ratio and cut-off value of the thyroid tissue was higher in patients with chronic autoimmune thyroiditis compared with the values in normal thyroid parenchyma in RTE, suggesting that these two diagnostic parameters may be helpful for diagnosis or follow-up of lymphoma and malign nodules (26). Furthermore, the diagnostic values of CEUS or RTE alone were previously assessed in the characterization of thyroid nodules (16). RTE may be useful in the diagnosis of thyroid nodules to exclude papillary thyroid cancer, but not follicular carcinoma; CEUS shows no improvement in the characterization of thyroid nodules (16). A previous study also revealed that Q-elastography is a better tool in differentiating the underlying nature of thyroid nodules compared with CEUS (27); however, a combination of CEUS and RTE in the differentiation of benign and malignant thyroid nodules requires to be further evaluated.

In the current study, the diagnostic values of CEUS alone, RTE alone, and a combination of CEUS and RTE in distinguishing benign from malignant thyroid nodules were examined.

\section{Materials and methods}

Subjects. Between August 2012 and June 2014, 97 patients (50 male and 47 female patients; mean age, $48.6 \pm 12.4$; age range, 27-70 years) scheduled for surgical removal of thyroid nodules were recruited at the Department of Gland Surgery. The patient inclusion criteria for this study were the following: i) Surgery indication for palpable or impalpable thyroid nodules; ii) patients scheduled for surgical removal of thyroid nodules; iii) the final diagnosis was confirmed by histopathologic examination of resected thyroid gland tissue; and iv) the patients did not suffer from any serious allergies. Histopathological changes were observed by hematoxylin and eosin (HE) staining. The study was approved by the Institutional Review Board of the Second and Third Hospital of Hebei Medical University. Written informed consent was obtained from all participants. The study protocols conformed to the Declaration of Helsinki (28).

CEUS. All patients were examined using a Philips IU22 US system equipped with a L12-5 wide frequency linear array probe (Philips Medical Systems, Inc., Bothell, WA, USA). Initially, patients were assessed in a routine US examination in supine position with the neck fully exposed. Thyroid nodules were evaluated for location, size, margin, internal echo, evenness and calcification. Contrast pulse sequencing was also applied, with probe emission frequency of 9-12 MHz, mechanical index of 0.08 , imaging depth of $50 \mathrm{~mm}, 50 \%$ image gain and US pressure of $50 \mathrm{kPa}$. SonoVue ${ }^{\circledR}$ (Bracco Imaging SpA, Milan, Italy) was used as US contrast agent. SonoVue ${ }^{\circledR}$ was dissolved in $5 \mathrm{ml} 0.9 \%$ sodium chloride and was injected as an intravenous bolus of $2.4 \mathrm{ml}$ per subject via an antecubital vein, followed by additional $5 \mathrm{ml} 0.9 \%$ sodium chloride. Subsequent to contrast agent injection, harmonic gray-scale CEUS was applied to scan the thyroid gland and the nodule for at least $150 \mathrm{sec}$, and the dynamic images were recorded.

RTE. RTE was performed with an Acuson S2000 system (Siemens Medical Solutions, Malvern, PA, USA), with a high frequency linear array probe $(6.5-13 \mathrm{MHz})$. With the patients in the supine position, the routine US examination was performed to locate the thyroid nodules and the ultrasonographic features of the focus were recorded. Subsequently, RTE was performed and the region-of-interest (ROI) was selected for the elastography examination (2-3 times bigger than the focus) with the probe vibrating 1-2 times/s, while the pressure indication on the screen was controlled within 3-4 kPa. The resultant elastogram was displayed over the B-mode image and the images were assessed by a color scale: Green indicated 'medium stiffness' of the tissue in the ROI, blue indicated 'harder tissue' and red indicated 'softer tissue'.

Image analysis and diagnosis criteria. The differential diagnosis of malignant or benign thyroid nodules was based on the results obtained from CEUS and RTE, and was performed by two experienced radiologists in a double blinded manner. Enhancement features of lesion on CEUS included: i) Margin enhancement, defined as clear or unclear based on the clarity of the margin between the lesion and peripheral tissue; ii) shape enhancement, classified as regular or irregular based on the shapes observed following contrast agent injection; iii) area enhancement, defined as $<50$ or $\geq 50 \%$ based on the area of the enhancement part/the lesion section at the peak enhancement; iv) enhancement degree, characterized as low, equal or high intensity when compared with the surrounding thyroid parenchyma; and v) type of enhancement, including homogeneous (relative homogeneous diffuse enhancement in lesions), heterogeneous (diffuse enhancement presenting inhomogeneous or regional microvesicle distribution) and ring enhancement (ring structure, such as round, oval or oblate spheroid). In CEUS, lesions that showed inhomogeneous and low enhancement were considered as diagnostic criteria for malignant nodules $(29,30)$. Elasticity was classified in five different patterns on RTE scans, as described previously $(31,32)$. These patterns include the following: classification I, in which the nodule is displayed homogeneously in green; classification II, in which the nodule is displayed predominantly in green with few red areas/spots; classification III, in which the nodule is displayed predominantly in red with few green areas/spots; classification IV, where the nodule is displayed homogeneously in red; and classification $\mathrm{V}$, where the nodule is displayed with mainly red areas. The elasticity of the thyroid nodules was observed using real-time elastography along with the corresponding contrast enhanced ultrasound images (Fig. 1). Nodules with elasticity III-V were suggested to be malignant thyroid nodules. The patients were diagnosed using the following CEUS and RTE combined method: CEUS and RTE-positive, malignancy was observed following both CEUS and RTE image analysis; either CEUS or 
A

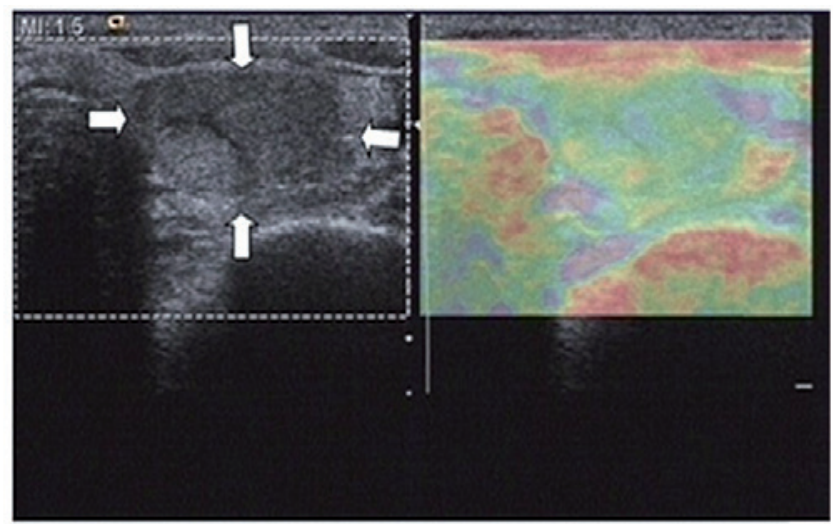

C

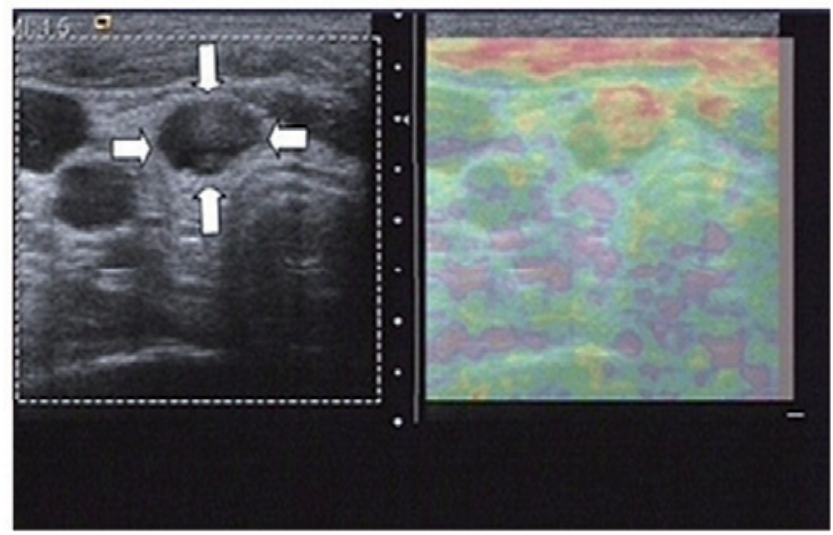

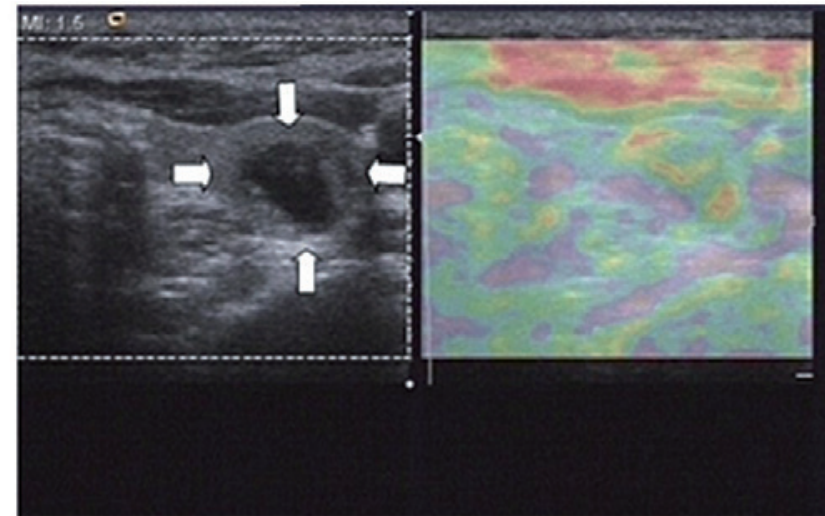

D

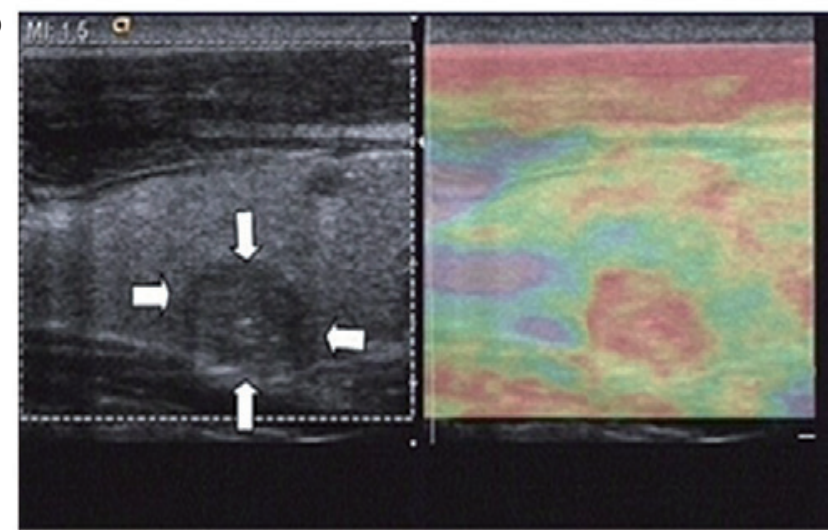

$\mathbf{E}$

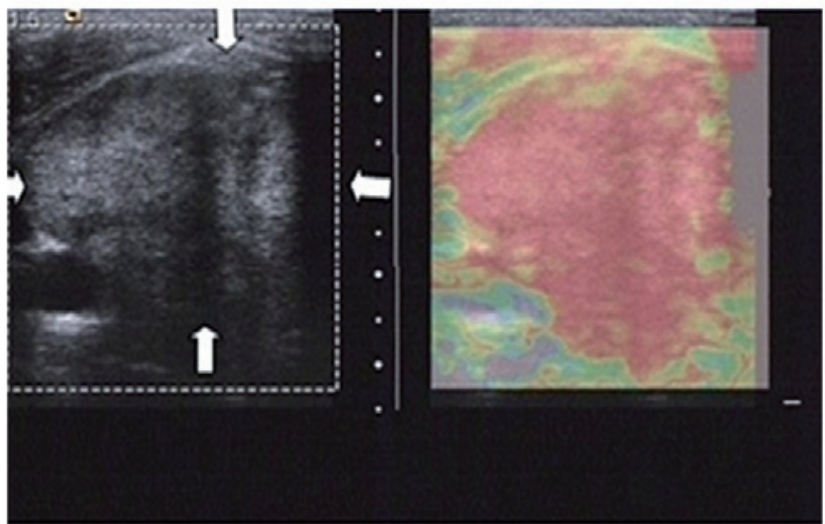

Figure 1. Elasticity of thyroid nodules, as observed using real time elastography, along with the corresponding contrast enhanced ultrasound images. The following elasticity classifications were observed: (A) I; (B) II; (C) III; (D) IV; and (E) V. Nodules with III-V elasticity were suggested to be malignant thyroid nodules.

RTE-positive, malignancy was observed following either CEUS or RTE image analysis; CEUS and RTE-negative, benign in both CEUS and RTE images.

Hematoxylin and eosin staining. Thyroid nodule tissue samples were obtained by surgery, and the the tissue samples obtained were fixed in $10 \%$ formaldehyde solution, dehydrated and embedded in paraffin. Each paraffin-embedded tissue sample was cut into 6 sections $(3 \mu \mathrm{m})$ using a microtome (1512-type Ultra-Thin Semiautomatic Microtome; Leica Microsystems $\mathrm{GmbH}$, Wetzlar, Germany). The tissue sections were then placed on glass slides, treated with $98 \%$ sulphuric acid, washed with distilled water, treated with $95 \%$ alcohol overnight, and coated with $1 \%$ poly-L-lysine (Beijing Solarbio Science \& Technology Co., Ltd., Beijing, China) following high temperature drying at $60^{\circ} \mathrm{C}$. Hematoxylin and eosin staining was performed after overnight oven treatment at $65^{\circ} \mathrm{C}$. Briefly, the tissue samples were dewaxed twice in xylene for $10 \mathrm{~min}$, rehydrated with graded ethanol ( $2 \mathrm{~min} /$ grade; 100, 95, 85 and 70\% ethanol) and washed for 2-3 min with distilled water. The tissue samples were then stained with hematoxylin for $5 \mathrm{~min}$ at room temperature, rinsed in running water, treated with $1 \%$ hydrochloric acid alcohol for $30 \mathrm{sec}$, and washed in running water for $15 \mathrm{~min}$ or warm water $\left(\sim 50^{\circ} \mathrm{C}\right)$ for $5 \mathrm{~min}$. The tissue samples were then stained with eosin for $2 \mathrm{~min}$ at room temperature, dehydrated with graded ethanol (70, 85, 95 and $100 \%$ ethanol), twice deparaffinized with xylene for $10 \mathrm{~min}$, and sealed with neutral gum. The results were observed and images of the tissue samples were captured using an Olympus CHK microscope imaging system (Olympus Corporation, Tokyo, Japan).

Statistical analysis. Continuous variables are presented as the mean \pm standard deviation, while categorical variables are presented as percentages of the total value. 
Table I. Characteristics of conventional ultrasound of benign and malignant thyroid nodules.

\begin{tabular}{|c|c|c|c|c|}
\hline Characteristic & Malignant $(\mathrm{n}=66)$ & Benign $(n=43)$ & $\chi^{2}$ & P-value \\
\hline Shape & & & 0.952 & 0.329 \\
\hline Regular & 29 & 23 & & \\
\hline Irregular & 37 & 20 & & \\
\hline Aspect ratio & & & 10.200 & 0.001 \\
\hline$<1$ & 32 & 34 & & \\
\hline$\geq 1$ & 34 & 9 & & \\
\hline Margin & & & 77.840 & $<0.001$ \\
\hline Well-defined, smooth & 9 & 43 & & \\
\hline Poorly-defined & 57 & 0 & & \\
\hline Echogenicity & & & 21.010 & $<0.001$ \\
\hline Hyperechogenicity & 17 & 30 & & \\
\hline Isoechogenicity & 24 & 8 & & \\
\hline Hypoechogenicity & 25 & 5 & & \\
\hline Echotexture & & & 0.333 & 0.564 \\
\hline Homogeneous & 27 & 20 & & \\
\hline Heterogeneous & 39 & 23 & & \\
\hline Calcification & & & 20.560 & $<0.001$ \\
\hline Microcalcification & 49 & 13 & & \\
\hline Macrocalcification & 17 & 30 & & \\
\hline
\end{tabular}

Comparisons between groups were performed using $\chi^{2}$ test and Student's t-test, as appropriate. Considering the postoperative pathological results as the golden standard, the sensitivity, specificity, positive predictive value (PPV), negative predictive value (NPV) and accuracy of CEUS alone, RTE alone and combination of CEUS and RTE were calculated. To characterize the diagnostic performance of RTE and CEUS, receiver operating characteristic (ROC) curve analyses were implemented and estimated by Hanley-McNeil non-parametric analysis. Statistical analysis was conducted using the SPSS version 20.0 software (IBM Corp., Armonk, NY, USA). $\mathrm{P}<0.05$ was considered to indicate a statistically significant difference.

\section{Results}

Pathological examination and US characteristics of thyroid nodules. Pathological examination of 109 nodules in the 97 patients (some patients had more than one nodule) showed 66 papillary carcinomas and 43 benign lesions (including 21 adenoma and 22 nodular goiters). In addition, among the 109 nodules, solitary nodules were observed in 14 cases and multiple nodules were observed in 83 cases. Conventional US of the 66 papillary carcinomas revealed 37 nodules with irregular shape, 34 nodules with a longitudinal/transverse diameter aspect ratio of $>1,39$ nodules with heterogeneous internal echoes and 49 nodules with microcalcifications (Fig. 2A). All papillary carcinoma nodules presented with a poorly-defined margin and marked hypoechogenicity, while the mean diameter of the nodules was $2.41 \pm 1.14 \mathrm{~cm}$.

Among the 43 benign lesions, conventional US showed 20 nodules with irregular shape, 9 nodules with an aspect ratio of $>1,30$ nodules with marked hyperechogenicity, 20 nodules with heterogeneous internal echoes, and 13 nodules with microcalcifications (Fig. 2B). In addition, all nodules presented with a poorly-defined margin and the mean size of the examined nodules was $2.32 \pm 1.04 \mathrm{~cm}$.

Significant differences were detected in the aspect ratio, margin, echogenicity and presence of calcifications (all $\mathrm{P}<0.05)$ between the malignant and benign tumors. However, no significant differences were identified in the lesion shape or echotexture (both $\mathrm{P}>0.05$; Table I). The results demonstrate that thyroid nodules with poorly defined tumors, aspect ratios $>1$ and microcalcifications are likely to be malignant (as shown in Table I). The histopathological images of the benign and malignant tumors are shown in Fig. 3.

CEUS characteristics of thyroid nodules. On CEUS scans, 40 out of the 43 benign nodules appeared to have well-defined margins, 38 nodules had a regular shape, 22 nodules showed an area of enhancement of $>50 \%$ and equal or high intensity, 4 nodules had lesions with heterogeneous enhancement, 26 nodules showed homogeneous enhancement, and 13 nodules presented with ring enhancement. The characteristics of representative examples of benign nodules on CEUS are presented in Fig. 4A. By contrast, out of the 66 malignant nodules, 15 nodules had lesions with well-defined margin, 15 nodules exhibited a regular shape, 50 nodules had area of enhancement of $>50 \%$ with equal or high intensity, 7 nodules had lesions with homogeneous enhancement, 53 heterogeneous enhancement and 6 ring enhancement. The characteristics of representative examples of malignant nodules on CEUS are presented in Fig. 4B. The findings showed statistically significant differences in the 
Table II. Characteristics of contrast-enhanced ultrasound of benign and malignant thyroid nodules.

\begin{tabular}{|c|c|c|c|c|}
\hline Enhancement characteristics & Malignant $(n=66)$ & Benign $(n=43)$ & $\chi^{2}$ & $\mathrm{P}$-value \\
\hline Margin & & & 51.47 & $<0.001$ \\
\hline Well-defined & 15 & 40 & & \\
\hline Poorly-defined & 51 & 3 & & \\
\hline Shape & & & 44.91 & $<0.001$ \\
\hline Regular & 15 & 38 & & \\
\hline Irregular & 51 & 5 & & \\
\hline Area & & & 7.024 & $<0.008$ \\
\hline$<50 \%$ & 16 & 21 & & \\
\hline$\geq 50 \%$ & 50 & 22 & & \\
\hline Intensity & & & 52.63 & $<0.001$ \\
\hline High & 7 & 20 & & \\
\hline Equal & 6 & 19 & & \\
\hline Low & 53 & 4 & & \\
\hline Type & & & 10.23 & 0.006 \\
\hline Heterogeneous & 53 & 4 & & \\
\hline Homogeneous & 7 & 26 & & \\
\hline Ring & 6 & 13 & & \\
\hline
\end{tabular}

A

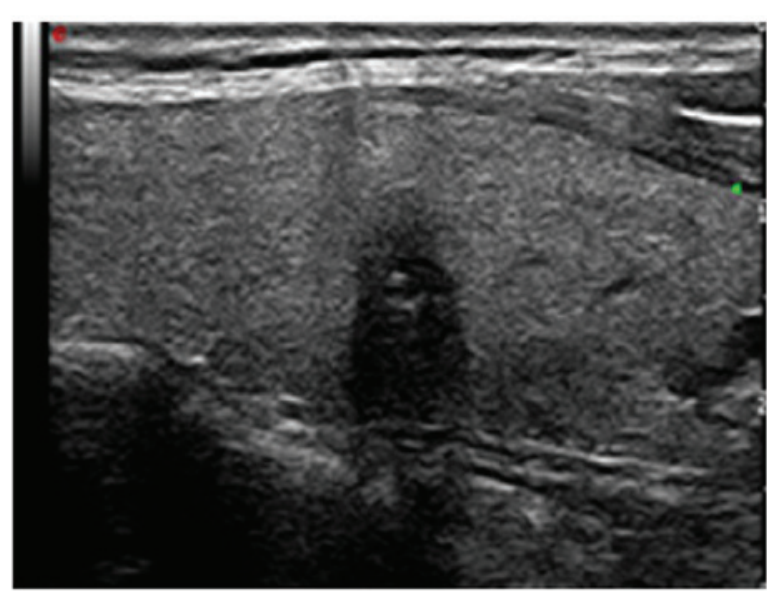

B

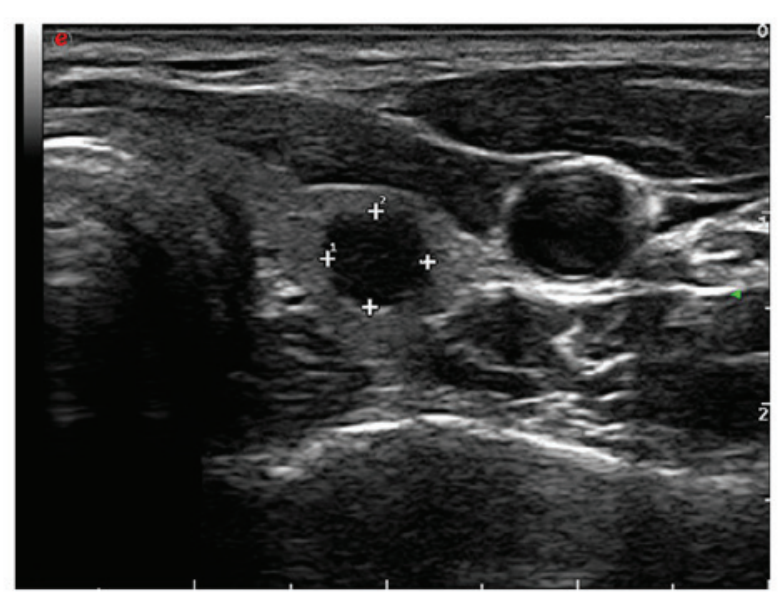

Figure 2. Representative examples of malignant and benign nodules using conventional ultrasound. (A) papillary carcinomas; (B) nodular goiters

enhancement margin, shape, enhancement area, intensity and type of enhancement between benign and malignant thyroid nodules (all $\mathrm{P}<0.05$; Table II).

RTE characteristics of thyroid nodules. As shown in Table III, in the 43 benign thyroid nodules, elasticity I was seen in 25 nodules, elasticity II in 13 nodules, elasticity III in 4 nodules, elasticity IV in 1 nodule, and no nodule exhibited an elasticity classification V. By contrast, in the 66 malignant thyroid nodules, there were 6 nodules in elasticity I, 5 nodules in elasticity II, 17 nodules in elasticity III, 25 nodules in elasticity IV, 13 nodules in elasticity V. The results indicated that significant differences existed in the elasticity classifications I, II, IV and V between malignant and benign nodules (all $\mathrm{P}<0.001$ ), whereas no difference was identified in elasticity classification III $(\mathrm{P}>0.05)$. These results suggest that benign tumors commonly exhibit lower elasticity grades, whereas elasticity grades III-V are predominantly observed in malignant nodules.

Diagnostic accuracy of CEUS alone, RTE alone and CEUS +RTE in thyroid nodules. The sensitivity and specificity of CEUS in differentiating benign and malignant thyroid nodules were 81.82 and $90.70 \%$, respectively. In addition, the PPV was 93.10 , the NPV was $90.70 \%$, and the accuracy was found to be $85.32 \%$. With respect to RTE, the sensitivity was $80.30 \%$, and the specificity was $88.37 \%$, while the PPV and NPV were 91.38 and $88.37 \%$, respectively, and the accuracy of RTE was $83.49 \%$. Combined diagnosis using CEUS and RTE had a sensitivity of $95.45 \%$, a specificity of $95.35 \%$, a PPV of 96.92, an NPV of 95.35\%, and an accuracy of $96.00 \%$. Compared with the CEUS and RTE alone, the combined RTE 
Table III. Elasticity imaging classification of benign and malignant thyroid nodules.

\begin{tabular}{lcrrr}
\hline Elasticity imaging classification & Malignant $(\mathrm{n}=66)$ & Benign $(\mathrm{n}=43)$ & $\chi^{2}$ & P-value \\
\hline I & 6 & 25 & 16.400 & $<0.001$ \\
II & 5 & 13 & 6.731 & 0.010 \\
III & 17 & 4 & 2.931 & 0.075 \\
IV & 25 & 1 & 12.110 & $<0.001$ \\
V & 13 & 0 & 7.920 & 0.005 \\
\hline
\end{tabular}

Table IV. Comparison of the sensitivity, specificity, PPV, NPV and accuracy of CEUS alone, RTE alone and combination of CEUS + RTE in the differential diagnosis of malignant and benign thyroid nodules.

\begin{tabular}{|c|c|c|c|c|c|c|c|}
\hline \multirow[b]{2}{*}{ Detection } & \multicolumn{2}{|c|}{ Pathological results } & \multirow[b]{2}{*}{ Sensitivity } & \multirow[b]{2}{*}{ Specificity } & \multirow[b]{2}{*}{ PPV } & \multirow[b]{2}{*}{ NPV } & \multirow[b]{2}{*}{ Accuracy } \\
\hline & $\begin{array}{l}\text { Malignant } \\
\quad(n=66)\end{array}$ & $\begin{array}{l}\text { Benign } \\
(n=43)\end{array}$ & & & & & \\
\hline CEUS & & & $81.82 \%$ & $90.70 \%$ & $93.10 \%$ & $90.70 \%$ & $85.32 \%$ \\
\hline Malignant & 54 & 4 & & & & & \\
\hline Benign & 12 & 39 & & & & & \\
\hline RTE & & & $80.30 \%$ & $88.37 \%$ & $91.38 \%$ & $88.37 \%$ & $83.49 \%$ \\
\hline Malignant & 53 & 5 & & & & & \\
\hline Benign & 13 & 38 & & & & & \\
\hline CEUS + RTE & & & $95.45 \%$ a,b & $95.35 \%$ a,b & $96.92 \%$ & $95.35 \%$ & $95.41 \%^{\mathrm{a}, \mathrm{b}}$ \\
\hline Positive (all/any) & 63 & 2 & & & & & \\
\hline Negative (all) & 3 & 41 & & & & & \\
\hline
\end{tabular}

${ }^{\mathrm{a} P}<0.05$, vs. CEUS; ${ }^{\mathrm{b}} \mathrm{P}<0.05$, vs. RTE. CEUS, contrast-enhanced ultrasound; RTE, real-time elastography; PPV, positive predictive value; NPV, negative predictive value.

Table V. Results of receiver operating characteristic (ROC) curve analysis for CEUS alone, RTE alone and combination of CEUS + RTE in the differential diagnosis of malignant and benign thyroid nodules.

\begin{tabular}{|c|c|c|c|c|c|}
\hline \multirow[b]{2}{*}{ Diagnostic approach } & \multirow[b]{2}{*}{ AUC } & \multirow[b]{2}{*}{ Standard error } & \multirow[b]{2}{*}{ P-value ${ }^{b}$} & \multicolumn{2}{|c|}{$95 \% \mathrm{CI}$} \\
\hline & & & & Upper limit & Lower limit \\
\hline CEUS & 0.883 & 0.037 & $<0.001$ & 0.810 & 0.956 \\
\hline RTE & 0.863 & 0.040 & $<0.001$ & 0.785 & 0.841 \\
\hline CEUS + RTE & $0.959^{\mathrm{a}}$ & 0.023 & $<0.001$ & 0.904 & 1.000 \\
\hline
\end{tabular}

${ }^{\mathrm{a}} \mathrm{P}<0.05$ vs. RTE; ${ }^{\mathrm{b}} \mathrm{P}<0.001$ vs. reference (AUC=0.5). CEUS, contrast-enhanced ultrasound; RTE, real-time elastography; AUC, area under the curve; $95 \%$ CI, $95 \%$ confidence interval.

and CEUS approach was associated with higher sensitivity and specificity for predicting benign and malignant thyroid nodules $(\mathrm{P}<0.05)$. Notably, there was no significant difference in the sensitivity, specificity, PPV, NPV and accuracy between CEUS and RTE ( $P>0.05$; Table IV).

Based on the results of ROC analysis, the area under the curve (AUC) was 0.883 [95\% confidence interval (CI), 0.810-0.956], 0.863 (95\% CI, 0.785-0.841) and 0.959 (95\% CI, 0.904-1.000) for CEUS, RTE and CEUS + RTE, respectively
(Table V). Statistically significant differences in AUC were detected between CEUS, RTE and CEUS + RTE, and the reference (AUC $=0.5$; all $\mathrm{P}<0.001$ ). Additionally, a significant difference in AUC was found between RTE and CEUS + RTE $(\mathrm{P}<0.05)$, while a similar association was not observed between CEUS and RTE, CEUS and CEUS+RT (all P>0.05; Table V). The ROC curve for CEUS, RTE and CEUS + RTE (Hanley-McNeil non-parametric analysis) for differentiation of thyroid nodules is shown in Fig. 5. 

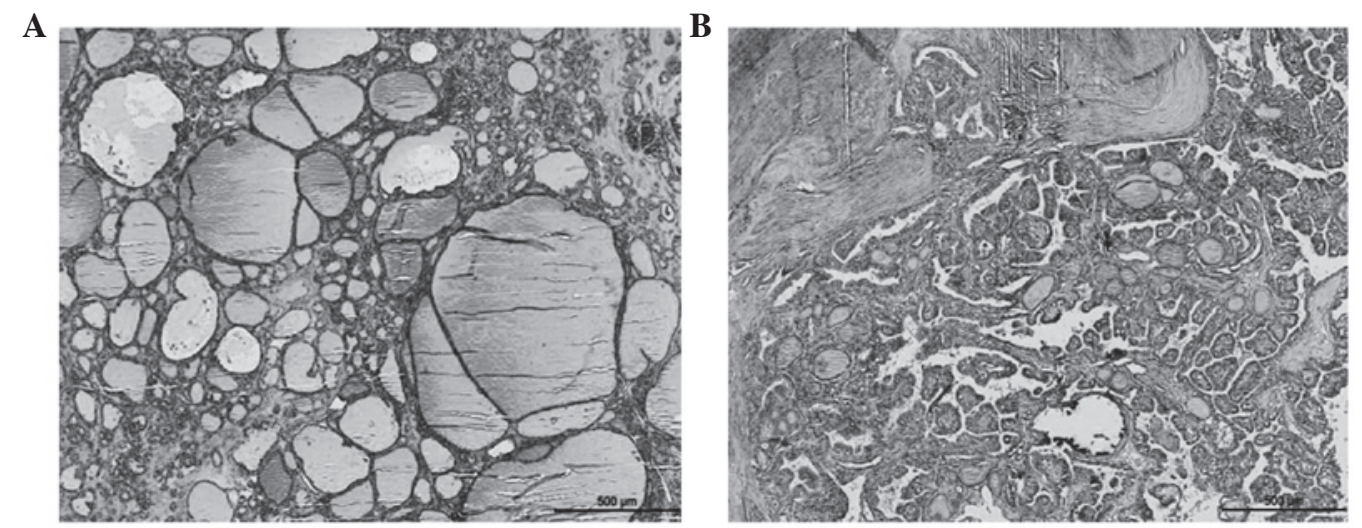

Figure 3. Representative examples of (A) benign (nodular goiters) and (B) malignant (papillary carcinomas) thyroid nodules, stained by hematoxylin-eosin (magnification, $\mathrm{x} 40$ ).

A

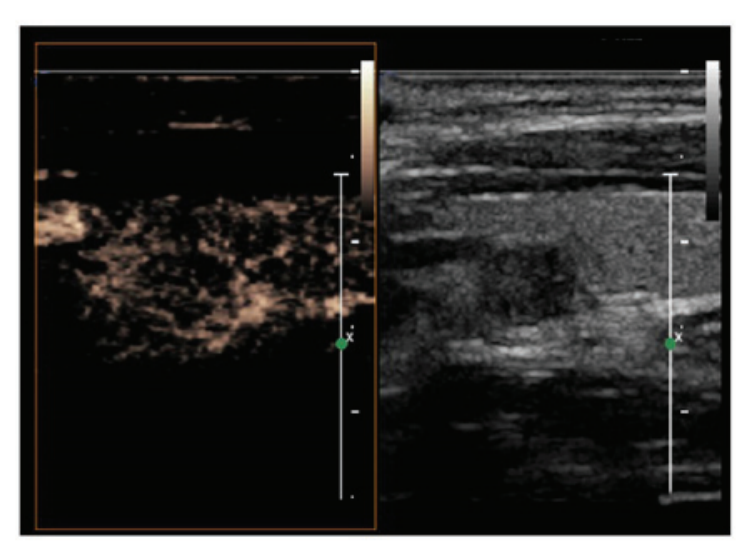

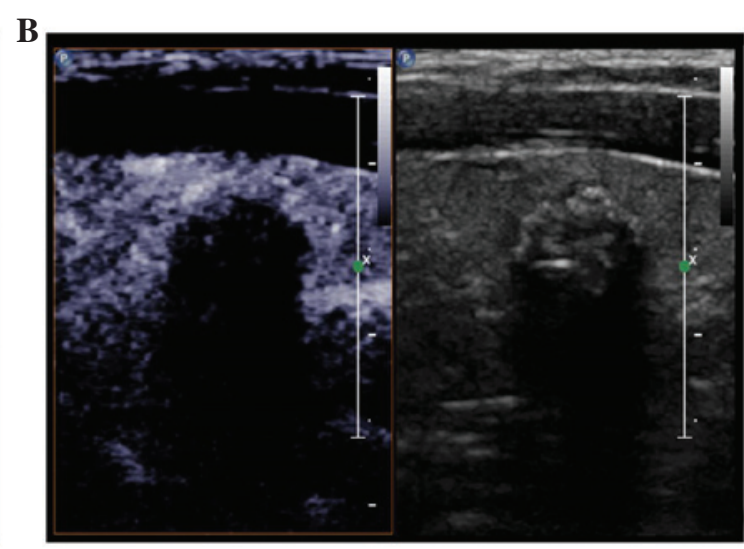

Figure 4. Characteristics of contrast-enhanced ultrasound of benign and malignant thyroid nodules. (A) Representative example of a benign nodular goiter, which appeared with a well-defined margin, regular shape, area of enhancement of $>50 \%$ and high intensity with heterogeneous enhancement. (B) Representative papillary carcinoma, showing a poorly-defined margin, irregular shape, area of enhancement of $>50 \%$ and equal intensity with heterogeneous enhancement.

\section{Discussion}

The present study evaluated the clinical value of CEUS and RTE, alone and in combination, in differentiating between benign and malignant thyroid nodules. To the best of our knowledge, this is the first such analysis to conclude that a combination of RTE and CEUS has a better diagnostic value for thyroid nodules. Significant findings in the present study demonstrated that CEUS + RTE displayed a higher sensitivity and specificity when compared with CEUS or RTE alone, indicating the high diagnostic performance of CEUS + RTE in distinguishing benign from malignant thyroid nodules.

The diagnostic performance of CEUS and RTE has been individually assessed by numerous studies (33-35). On CEUS scans, papillary thyroid carcinoma shows a heterogeneous low enhancement pattern, whereas nodular goiters show an equal enhancement pattern (28). The detection of papillary thyroid carcinomas with low enhancement has a sensitivity of $96.8 \%$, specificity of $95.0 \%$ and accuracy of $95.9 \%$ (36). CEUS has been demonstrated to have a high diagnostic value in the differential diagnosis of benign and malignant calcified thyroid nodules, with sensitivity, specificity, PPV, NPV and accuracy of $92.75,90.91,86.49,95.24$ and $91.62 \%$, respectively (30). A previous meta-analysis assessed the accuracy of CEUS in diagnosing thyroid nodules and the pooled sensitivity,

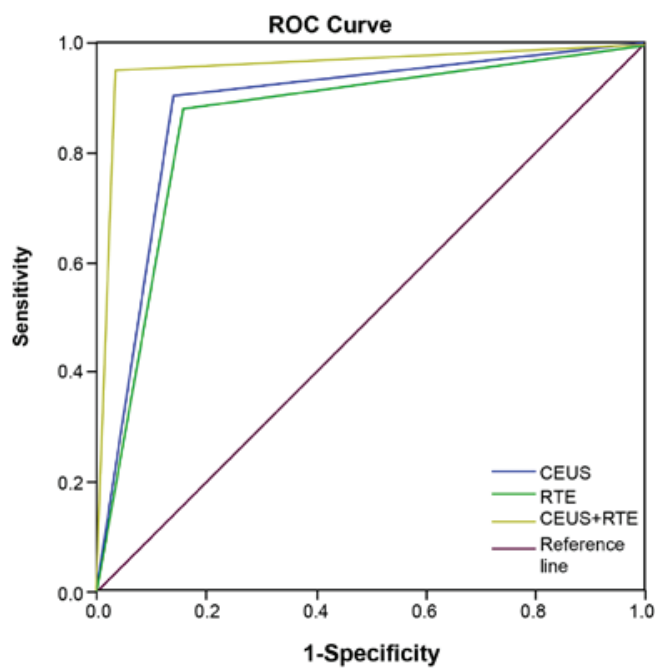

Figure 5. ROC curves for CEUS, RTE and CEUS + RTE in the diagnosis of benign and malignant thyroid nodules. ROC, receiver operating characteristic; CEUS, contrast-enhanced ultrasound; RTE, real-time elastography.

specificity, and positive and negative likelihood ratios were found to be $0.853,0.876,5.822$ and 0.195 , respectively (19). In addition, the diagnostic odds ratio and AUC were 34.730 and 
0.9162, respectively (19). It has been suggested that RTE has a high sensitivity and specificity for the diagnosis of malignant thyroid nodules, with an overall mean sensitivity and specificity of 92\% (95\% CI, 88-96) and 90\% (95\%CI, 85-95), respectively (37). This demonstrated that RTE may be useful in conjunction or even instead of fine-needle aspiration biopsy for the selection of patients with thyroid nodules requiring surgery (37). Furthermore, according to semiquantitative elastosonography, the sensitivity, specificity, PPV and NPV were 95, 88, 97 and 91\% respectively, whereas in CEUS, the sensitivity, specificity, PPV and NPV were 79, 91, 83 and $89 \%$ respectively (22). CEUS and quantitative-elastosonography are both more specific when compared with US, while semiquantitative elastosonography seems to be more sensitive than CEUS (27). RTE is a promising diagnostic method that may be applied with a high NPV in the investigation of thyroid nodules in order to exclude papillary thyroid carcinoma, while CEUS with SonoVue ${ }^{\circledR}$ have not been found to improve the characterization of thyroid nodules (16).

In the current study, the margin, shape, area, intensity and type of enhancement presented with evident differences between benign and malignant thyroid nodules on CEUS scans. The sensitivity and specificity of CEUS in the differentiation between benign and malignant thyroid nodules were 81.82 and $90.70 \%$, respectively, with PPV and NPV of 93.10 and $90.70 \%$, respectively, while the accuracy was found to be $85.32 \%$. As for RTE, the elasticity classifications I, II, IV and V observed in the scans differed between benign and malignant thyroid nodules. The sensitivity was $80.30 \%$, the specificity was $88.37 \%$, PPV was 91.38 and NPV was $88.37 \%$, while the accuracy of RTE was $83.49 \%$. No significant difference was found in the diagnostic performance of CEUS or RTE alone for distinguishing benign from malignant thyroid nodules. CEUS + RTE presented with higher sensitivity and specificity when compared with CEUS or RTE alone in differentiating between the nodules. This may be due to CEUS + RTE providing noninvasive imaging of the mechanical characteristics of tissues, along with the use of a contrast medium providing an indirect description of intra-nodular vascularisation $(38,39)$. RTE conducted during endoscopic ultrasound allows the evaluation of tissue stiffness for better characterization of the lesions (39). In a previous study, liver metastases were not visible on conventional US, while multiple metastases were observed with the application of contrast agents, which indicates the usefulness of CEUS in the prediction of liver metastases (40). In addition, RTE has been suggested to be a useful tool for detecting lymph node metastases, which have been proven to be useful prognostic factors for papillary thyroid carcinoma (41-43). Therefore, the potential role of CEUS + RTE in predicting the tumor aggressiveness of thyroid nodules should be further assessed.

In conclusion, based on the results reported in the present study, the combination of CEUS + RTE may be the most efficient and effective diagnostic method for differential diagnosis of malignant and benign thyroid nodules. Thus, we present a potential improvement in the noninvasive diagnostic approach currently used, which may lead to successful implementation in accurate diagnosis and treatment of thyroid nodules. Further studies are required in order to further investigate the role of CEUS and RTE combined in the differential diagnosis of malignant and benign thyroid nodules.

\section{References}

1. Dean DS and Gharib H: Epidemiology of thyroid nodules. Best Pract Res Clin Endocrinol Metab 22: 901-911, 2008.

2. La Vecchia C, Malvezzi M, Bosetti C, Garavello W, Bertuccio P, Levi F and Negri E: Thyroid cancer mortality and incidence: A global overview. Int J Cancer 136: 2187-2195, 2015.

3. Vigneri R, Malandrino P and Vigneri P: The changing epidemiology of thyroid cancer: Why is incidence increasing? Curr Opin Oncol 27: 1-7, 2015.

4. Lozano R, Naghavi M, Foreman K, Lim S, Shibuya K, Aboyans V, Abraham J, Adair T, Aggarwal R, Ahn SY, et al: Global and regional mortality from 235 causes of death for 20 age groups in 1990 and 2010: A systematic analysis for the Global Burden of Disease Study 2010. Lancet 380: 2095-2128, 2012.

5. Aschebrook-Kilfoy B, Ward MH, Sabra MM and Devesa SS: Thyroid cancer incidence patterns in the United States by histologic type, 1992-2006. Thyroid 21: 125-134, 2011.

6. Chen W, Zheng R, Zeng H, Zhang S and He J: Annual report on status of cancer in China, 2011. Chin J Cancer Res 27: 2-12, 2015.

7. Wang Y and Wang W: Increasing incidence of thyroid cancer in Shanghai, China, 1983-2007. Asia Pac J Public Health 27: NP223-NP229, 2015.

8. Morris LF, Ragavendra N and Yeh MW: Evidence-based assessment of the role of ultrasonography in the management of benign thyroid nodules. World J Surg 32: 1253-1263, 2008.

9. Tamsel S, Demirpolat G, Erdogan M, Nart D, Karadeniz M, Uluer $\mathrm{H}$ and Ozgen AG: Power Doppler US patterns of vascularity and spectral Doppler US parameters in predicting malignancy in thyroid nodules. Clin Radiol 62: 245-251, 2007.

10. Kim JY, Lee CH, Kim SY, Jeon WK, Kang JH, An SK and Jun WS: Radiologic and pathologic findings of nonpalpable thyroid carcinomas detected by ultrasonography in a medical screening center. J Ultrasound Med 27: 215-223, 2008.

11. Algin O, Algin E, Gokalp G, Ocakoğlu G, Erdoğan C, Saraydaroglu O and Tuncel E: Role of duplex power Doppler ultrasound in differentiation between malignant and benign thyroid nodules. Korean J Radiol 11: 594-602, 2010.

12. Iannuccilli JD, Cronan JJ and Monchik JM: Risk for malignancy of thyroid nodules as assessed by sonographic criteria: The need for biopsy. J Ultrasound Med 23: 1455-1464, 2004.

13. Paschke R, Schmid KW, Gartner R, Mann K, Dralle H and Reiners C: Epidemiology, pathophysiology, guideline-adjusted diagnostics and treatment of thyroid nodules. Med Klin (Munich) 105: 80-87, 2010.

14. Russ G, Leboulleux S, Leenhardt L and Hegedüs L: Thyroid incidentalomas: Epidemiology, risk stratification with ultrasound and workup. Eur Thyroid J 3: 154-163, 2014.

15. Moon WJ, Jung SL, Lee JH, Na DG, Baek JH, Lee YH, Kim J, Kim HS, Byun JS, Lee DH, et al: Benign and malignant thyroid nodules: US differentiation-multicenter retrospective study. Radiology 247: 762-770, 2008.

16. Friedrich-Rust M, Sperber A, Holzer K, Diener J, Grünwald F, Badenhoop K, Weber S, Kriener S, Herrmann E, Bechstein WO, et al: Real-time elastography and contrast-enhanced ultrasound for the assessment of thyroid nodules. Exp Clin Endocrinol Diabetes 118: 602-609, 2010.

17. Wang Y, Dan HJ, Dan HY, Li T and Hu B: Differential diagnosis of small single solid thyroid nodules using real-time ultrasound elastography. J Int Med Res 38: 466-472, 2010.

18. Minami Y and Kudo M: Ultrasound fusion imaging of hepatocellular carcinoma: A review of current evidence. Dig Dis 32: 690-695, 2014.

19. Yu D, Han Y and Chen T: Contrast-enhanced ultrasound for differentiation of benign and malignant thyroid lesions: Meta-analysis. Otolaryngol Head Neck Surg 151: 909-915, 2014.

20. Jiang J, Huang L, Zhang H, Ma W, Shang X, Zhou Q, Gao Y, Yu S and Qi Y: Contrast-enhanced sonography of thyroid nodules. J Clin Ultrasound 43: 153-156, 2015.

21. Nemec U, Nemec SF, Novotny C, Weber M, Czerny C and Krestan CR: Quantitative evaluation of contrast-enhanced ultrasound after intravenous administration of a microbubble contrast agent for differentiation of benign and malignant thyroid nodules: Assessment of diagnostic accuracy. Eur Radiol 22: 1357-1365, 2012.

22. Agha A, Jung EM, Janke M, Hornung M, Georgieva M, Schlitt HJ, Schreyer AG, Strosczcynski C and Schleder S: Preoperative diagnosis of thyroid adenomas using high resolution contrast-enhanced ultrasound (CEUS). Clin Hemorheol Microcirc 55: 403-409, 2013. 
23. Deng J, Zhou P, Tian SM, Zhang L, Li JL and Qian Y: Comparison of diagnostic efficacy of contrast-enhanced ultrasound, acoustic radiation force impulse imaging and their combined use in differentiating focal solid thyroid nodules. PLoS One 9: e90674, 2014.

24. Kwak JY and Kim EK: Ultrasound elastography for thyroid nodules: Recent advances. Ultrasonography 33: 75-82, 2014.

25. Şahin M, Çakal E, Özbek M, Güngünes A, Arslan MS Akkaymak ET, Uçan B, Ünsal IÖ, Bozkurt NC and Delibaşi T: Elastography in the differential diagnosis of thyroid nodules in Hashimoto thyroiditis. Med Oncol 31: 97, 2014.

26. Menzilcioglu MS, Duymus M, Gungor G, Citil S, Sahin T, Boysan SN and Sarica A: The value of real-time ultrasound elastography in chronic autoimmune thyroiditis. Br J Radiol 87: 20140604, 2014.

27. Cantisani V, Consorti F, Guerrisi A, Guerrisi I, Ricci P, Di Segni M, Mancuso E, Scardella L, Milazzo F, D'Ambrosio F and Antonaci A: Prospective comparative evaluation of quantitative-elastosonography (Q-elastography) and contrast-enhanced ultrasound for the evaluation of thyroid nodules: Preliminary experience. Eur J Radiol 82: 1892-1898, 2013.

28. M PN: World Medical Association publishes the Revised Declaration of Helsinki. Natl Med J India 27: 56, 2014.

29. Zhang B, Jiang YX, Liu JB, Yang M, Dai Q, Zhu QL and Gao P: Utility of contrast-enhanced ultrasound for evaluation of thyroid nodules. Thyroid 20: 51-57, 2010.

30. Zhou Q, Xu YB, Jiang J, Ma WQ, Wang H, Li M and Lei XY: Differential diagnostic value of contrast-enhanced ultrasound in calcified thyroid nodules. Zhonghua Er Bi Yan Hou Tou Jing Wai Ke Za Zhi 48: 726-729, 2013 (In Chinese).

31. Yuan WH, Chiou HJ, Chou YH, Hsu HC, Tiu CM, Cheng CY and Lee $\mathrm{CH}$ : Gray-scale and color Doppler ultrasonographic manifestations of papillary thyroid carcinoma: Analysis of 51 cases. Clin Imaging 30: 394-401, 2006.

32. Stacul F, Bertolotto M, De Gobbis F, Calderan L, Cioffi V, Romano A, Zanconati F and Cova MA: US, colour-Doppler US and fine-needle aspiration biopsy in the diagnosis of thyroid nodules. Radiol Med 112: 751-762, 2007.

33. Patel KV1, Huang DY1, Sidhu PS1. Metachronous bilateral segmental testicular infarction: multi-parametric ultrasound imaging with grey-scale ultrasound, Doppler ultrasound, contrast-enhanced ultrasound (CEUS) and real-time tissue elastography (RTE). J Ultrasound. 2014 May 31;17(3):233-8
34. Zheng XJ, Zhang YK, Zhao CY, Shi XL, Li CS, Jiang JF, Wang $\mathrm{H}$ and $\mathrm{Ye} \mathrm{B}$ : Diagnosis of thyroid space-occupying lesions using real-time contrast-enhanced ultrasonography with sulphur hexafluoride microbubbles. Chinese Journal of Otorhinolaryngology Head and Neck Surgery 44: 277-281, 2009 (In Chinese).

35. Frey H: Realtime elastography. A new ultrasound procedure for the reconstruction of tissue elasticity. Radiologe 43: 850-855, 2003 (In German)

36. Jiang J, Shang X, Zhang H, Ma W, Xu Y, Zhou Q, Gao Y, Yu S and Qi Y: Correlation between maximum intensity and microvessel density for differentiation of malignant from benign thyroid nodules on contrast-enhanced sonography. J Ultrasound Med 33: 1257-1263, 2014.

37. Bojunga J, Herrmann E, Meyer G, Weber S, Zeuzem S and Friedrich-Rust M: Real-time elastography for the differentiation of benign and malignant thyroid nodules: A meta-analysis. Thyroid 20: 1145-1150, 2010

38. Argalia G, De Bernardis S, Mariani D, Abbattista T, Taccaliti A, Ricciardelli L, Faragona S, Gusella PM and Giuseppetti GM: Ultrasonographic contrast agent: Evaluation of time-intensity curves in the characterisation of solitary thyroid nodules. Radiol Med 103: 407-413, 2002 (In English, Italian).

39. Dietrich CF, Saftoiu A and Jenssen C: Real time elastography endoscopic ultrasound (RTE-EUS), a comprehensive review. Eur J Radiol 83: 405-414, 2014.

40. Lorentzen T and Skjoldbye B: Diagnosis and biopsy of liver metastases using ultrasound contrast agent. Ugeskr Laeger 171: 907, 2009 (In Danish)

41. Aoyagi S, Izumi K, Hata H, Kawasaki H and Shimizu H: Usefulness of real-time tissue elastography for detecting lymph-node metastases in squamous cell carcinoma. Clin Exp Dermatol 34: e744-e747, 2009.

42. Sugitani I, Kasai N, Fujimoto Y and Yanagisawa A: A novel classification system for patients with PTC: Addition of the new variables of large ( $3 \mathrm{~cm}$ or greater) nodal metastases and reclassification during the follow-up period. Surgery 135: 139-148, 2004.

43. Sugitani I, Fujimoto Y, Yamada K and Yamamoto N: Prospective outcomes of selective lymph node dissection for papillary thyroid carcinoma based on preoperative ultrasonography. World J Surg 32: 2494-2502, 2008. 